\title{
Wiedemann-Franz law and nonvanishing temperature scale across the field-tuned quantum critical point of $\mathrm{YbRh}_{2} \mathrm{Si}_{2}$
}

\author{
J.-Ph. Reid ${ }^{1,}{ }^{*}$ M. A. Tanatar, ${ }^{1,2}$ R. Daou,,${ }^{1, \dagger}$ Rongwei Hu ${ }^{3, \ddagger}$ C. Petrovic, ${ }^{3,4}$ and Louis Taillefer ${ }^{1,4, \S}$ \\ ${ }^{1}$ Département de physique \& RQMP, Université de Sherbrooke, Sherbrooke, Québec, Canada JIK $2 R 1$ \\ ${ }^{2}$ Ames Laboratory and Department of Physics and Astronomy, Iowa State University, Ames, Iowa 50011, USA \\ ${ }^{3}$ Department of Physics, Brookhaven National Laboratory, Upton, New York 11973, USA \\ ${ }^{4}$ Canadian Institute for Advanced Research, Toronto, Ontario, Canada M5G 128
}

(Received 24 September 2013; revised manuscript received 17 December 2013; published 23 January 2014)

\begin{abstract}
The in-plane thermal conductivity $\kappa$ and electrical resistivity $\rho$ of the heavy-fermion metal $\mathrm{YbRh}_{2} \mathrm{Si}_{2}$ were measured down to $50 \mathrm{mK}$ for magnetic fields $H$ parallel and perpendicular to the tetragonal $c$ axis, through the field-tuned quantum critical point $H_{c}$, at which antiferromagnetic order ends. The thermal and electrical resistivities, $w \equiv L_{0} T / \kappa$ and $\rho$, show a linear temperature dependence below $1 \mathrm{~K}$, typical of the non-Fermi-liquid behavior found near antiferromagnetic quantum critical points, but this dependence does not persist down to $T=0$. Below a characteristic temperature $T^{\star} \simeq 0.35 \mathrm{~K}$, which depends weakly on $H, w(T)$ and $\rho(T)$ both deviate downward and converge as $T \rightarrow 0$. We propose that $T^{\star}$ marks the onset of short-range magnetic correlations, persisting beyond $H_{c}$. By comparing samples of different purity, we conclude that the Wiedemann-Franz law holds in $\mathrm{YbRh}_{2} \mathrm{Si}_{2}$, even at $H_{c}$, implying that no fundamental breakdown of quasiparticle behavior occurs in this material. The overall phenomenology of heat and charge transport in $\mathrm{YbRh}_{2} \mathrm{Si}_{2}$ is similar to that observed in the heavy-fermion metal $\mathrm{CeCoIn}_{5}$, near its own field-tuned quantum critical point.
\end{abstract}

DOI: 10.1103/PhysRevB.89.045130

\section{INTRODUCTION}

Quantum criticality has emerged as a central paradigm in the physics of heavy-fermion materials [1,2]. Spin fluctuations near a magnetic quantum critical point (QCP) lead to unusual electronic properties, deviating from those expected in the standard Fermi-liquid theory of metals. These deviations, called "non-Fermi-liquid (NFL) behavior," include a linear temperature dependence of the electrical resistivity $\rho$, in contrast to the expected $T^{2}$ behavior, and a logarithmic divergence of the specific heat $\gamma \equiv C / T$, as opposed to a constant $\gamma$, as $T \rightarrow 0$. A more profound form of NFL behavior would be a violation of the Wiedemann-Franz (WF) law [3-8], a robust property of charged fermions. This law states that the (Lorenz) ratio of the thermal conductivity $\kappa$ of a metal to its electrical conductivity $\sigma=1 / \rho$ has a universal value in the $T=0$ limit:

$$
L \equiv \frac{\kappa}{\sigma T}=L_{0}
$$

where $L_{0} \equiv\left(\pi^{2} / 3\right)\left(k_{\mathrm{B}} / e\right)^{2}$. Defining the thermal resistivity as $w \equiv L_{0} T / \kappa$, the WF law may be written as $w=\rho$ at $T=0$, or $\delta(0)=0$, where $\delta(T) \equiv w(T)-\rho(T)$ is the difference between heat and charge resistivities.

In the heavy-fermion metal $\mathrm{CeCoIn}_{5}$, a QCP is reached by tuning the magnetic field $H$ to $H_{c}=5.3 \mathrm{~T}(H \| c)[9,10]$. At $H_{c}, w(T)$ and $\rho(T)$ exhibit a linear $T$ dependence at low $T$,

\footnotetext{
*Present address: School of Physics and Astronomy, University of St Andrews, St Andrews KY16 9SS, UK.

${ }^{\dagger}$ Present address: Laboratoire CRISMAT, CNRS, Caen, France.

${ }_{\ddagger}^{\ddagger}$ Present address: Rutgers Center for Emergent Materials and Department of Physics and Astronomy, Rutgers University, Piscataway, New Jersey 08854, USA.

§Louis.Taillefer@USherbrooke.ca
}

PACS number(s): 71.10.Hf, 71.27.+a, 72.15.-v, 42.50.Lc

and so does $\delta(T)$ [11,12]. For currents in the basal plane of the tetragonal structure $(J \perp c), \delta(T)$ deviates downward from its linear $T$ dependence below $T \simeq 0.4 \mathrm{~K}$, and $\delta(T) \rightarrow 0$ as $T \rightarrow 0$ [11]. For currents normal to the basal plane $(J \| c)$, however, the linear $T$ dependence of $\delta(T)$ persists down to the lowest temperatures $(\sim 50 \mathrm{mK})$, and $\delta(T)$ extrapolates to a finite value at $T=0$ [12]. In other words, $\mathrm{CeCoIn}_{5}$ exhibits an anisotropic violation of the WF law.

This suggests that a linear $T$ dependence of $\rho(T)$ may be a prerequisite for the violation of the WF law at a QCP. This would be consistent with two previous studies, in which the WF law was found to be valid at the QCP, namely in the heavy-fermion metal $\mathrm{CeNi}_{2} \mathrm{Ge}_{2}$ (Ref. [13]) and in the ruthenate $\mathrm{Sr}_{3} \mathrm{Ru}_{2} \mathrm{O}_{7}$ [14]. Indeed, in neither case is $\rho(T)$ linear down to $T=0$. An exception may be found in the heavy-fermion metal $\mathrm{YbAgGe}$, for which a small violation has been reported, whereby $L / L_{0}=0.92 \pm 0.03$, even though the temperature dependence of $\rho(T)$ is not linear at the QCP [15].

It is of interest to investigate the WF law in other quantum critical systems. The heavy-fermion metal $\mathrm{YbRh}_{2} \mathrm{Si}_{2}$ is an ideal candidate for such a study. In zero field, it orders antiferromagnetically below a Néel temperature $T_{\mathrm{N}} \simeq 70 \mathrm{mK}$, and a small magnetic field suppresses $T_{\mathrm{N}}$ to zero, producing a field-tuned QCP at $H_{c}=0.66 \mathrm{~T}$ for $H \| c$, and at $H_{c}=0.06 \mathrm{~T}$ for $H \perp c$ [16,17], where $c$ is the [001] direction of the tetragonal crystal structure. NFL behavior is observed in $\mathrm{YbRh}_{2} \mathrm{Si}_{2}$, for example, as a linear $T$ dependence of the resistivity $(\rho \propto T)$ and a logarithmic $T$ dependence of the specific heat $(C / T \propto$ $\ln T$ ), for $H$ near $H_{c}$ [16,17]. It was suggested that local critical fluctuations [18] in this material make the entire Fermi surface "hot" and cause a breakdown of quasiparticles [17], which could produce a violation of the WF law.

Two recent reports provide conflicting interpretations on the validity of the WF law in $\mathrm{YbRh}_{2} \mathrm{Si}_{2}[19,20]$. The data by Pfau et al. [19], with $H \perp c$, show that, at $H_{c}, w \simeq \rho$ at the lowest measured temperature $(\sim 30 \mathrm{mK})$. However, the authors argue 
TABLE I. Zero-field characteristics of the $\mathrm{YbRh}_{2} \mathrm{Si}_{2}$ samples used to test the Wiedemann-Franz law. The residual resistivity ratio is defined as the ratio of resistance at room temperature $(300 \mathrm{~K})$ to resistance extrapolated to $T=0\left(\rho_{0}\right): \mathrm{RRR} \equiv \rho(300 \mathrm{~K}) / \rho_{0}$. To remove the uncertainty on $\rho_{0}$ that comes from the geometric factor, we set $\rho(300 \mathrm{~K})=80 \mu \Omega \mathrm{cm}$.

\begin{tabular}{lcc}
\hline \hline Sample & RRR & $\begin{array}{c}\rho_{0} \\
(\mu \Omega \mathrm{cm})\end{array}$ \\
\hline Pfau et al. [19] No. 1 & 50 & 1.6 \\
Pfau et al. [19] No. 2 & 73 & 1.1 \\
Machida et al. [20] & 90 & 0.9 \\
A & 105 & 0.75 \\
B & 120 & 0.66 \\
\hline \hline
\end{tabular}

that a contribution to heat transport from paramagnons must be subtracted from the measured $\kappa$, and this implies that the purely electronic $\delta(T)$ remains finite as $T \rightarrow 0$, so that the WF law is violated at $H_{c}$. The data by Machida et al. [20], with $H \| c$, show that, at $H_{c}, w(T) \rightarrow \rho(T)$ as $T \rightarrow 0$. Here, the authors argue that the WF law is in fact satisfied at $H_{c}$.

In this article, we report measurements of the electrical resistivity and thermal conductivity of $\mathrm{YbRh}_{2} \mathrm{Si}_{2}$, performed on high-quality single crystals for both field orientations. Both $w(T)$ and $\rho(T)$ exhibit a linear temperature dependence below $1 \mathrm{~K}$, but this dependence does not persist down to $50 \mathrm{mK}$. Even at the critical field $H_{c}$, it ends at a temperature $T^{\star} \simeq 0.35 \mathrm{~K}$. Below $T^{\star}$, both $w(T)$ and $\rho(T)$ deviate downward and start to converge. Because a similar, albeit sharper, drop in (and convergence of) $w(T)$ and $\rho(T)$ occurs below the antiferromagnetic ordering temperature $T_{\mathrm{N}}$ in $\mathrm{YbRh}_{2} \mathrm{Si}_{2}$ at $H=0$ and in the antiferromagnetic heavy-fermion metal CeRhIn ${ }_{5}$ [21], we propose that $T^{\star}$ marks the onset of short-range magnetic correlations.

Comparing data at $H_{c}$ from samples of different $\rho_{0}$ value, we conclude that the WF law is satisfied. As we shall show, the overall behavior of in-plane transport in $\mathrm{YbRh}_{2} \mathrm{Si}_{2}$ is similar to that of in-plane transport in $\mathrm{CeCoIn}_{5}$.

\section{EXPERIMENTAL}

High-quality single crystals of $\mathrm{YbRh}_{2} \mathrm{Si}_{2}$ were grown by the In flux method with a molar ratio of $\mathrm{YbRh}_{2} \mathrm{Si}_{2}: \mathrm{In}=5: 95$. Our method is similar to that of Ref. [22], but without the use of tantalum tubes. Starting ingredients were mixed in an alumina crucible and sealed into a quartz tube. The quartz tube was heated to $1150{ }^{\circ} \mathrm{C}$, held constant for 2 hours, and then cooled to $800{ }^{\circ} \mathrm{C}$, where crystals were decanted. The platelet crystals had dimensions up to $3 \times 3 \times 0.2 \mathrm{~mm}^{3}$ and were of high purity, as confirmed by their high residual resistivity ratio (RRR). Two samples, labeled A and B, were cut for electrical resistivity and thermal conductivity measurements, with their long side parallel to the [100] crystallographic direction ( $a$ axis), for a length of 1.5-2 mm and cross section of $0.1 \mathrm{~mm} \times 0.1 \mathrm{~mm}$. Four contacts were made on each sample by soldering silver wires with a silver-based alloy, giving a contact resistance of 1-2 $\mathrm{m} \Omega$ at low temperature. The samples have $\mathrm{RRR}=105$ (sample A) and 120 (sample B), slightly higher than the RRR of crystals used in the two previous studies[19,20] of the WF law in $\mathrm{YbRh}_{2} \mathrm{Si}_{2}$ (see Table I), but slightly lower than the

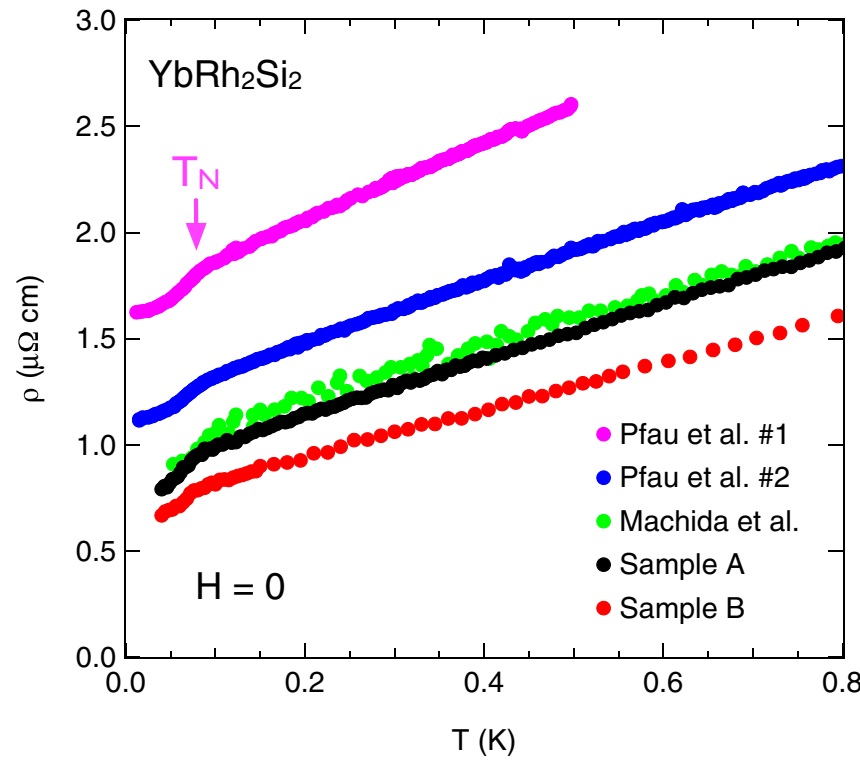

FIG. 1. (Color online) Temperature dependence of the in-plane electrical resistivity of five high-purity single crystals of $\mathrm{YbRh}_{2} \mathrm{Si}_{2}$, in zero field. Data for the two samples used in this study (sample A, black; sample B, red) are compared to previous data, from Pfau et al. (Ref. [19]) (No. 1, cyan; No. 2, blue) and Machida et al. (Ref. [20]) (green). The onset of antiferromagnetic order at $T_{\mathrm{N}}$ (arrow) is seen to cause a distinct drop in $\rho(T)$.

highest value of $\sim 150$ reported so far [17,23,24]. In Fig. 1, the zero-field $\rho(T)$ of both samples is compared to the data of Refs. [19] and [20]. All data are in good agreement, modulo a rigid shift due to the different $\rho_{0}$ values.

Thermal conductivity was measured using the same four contacts as in the four-probe resistivity measurement, in a standard one-heater-two-thermometers technique [25]. By using the same contacts, the relative uncertainty between heat and charge transport measurements is removed, and a precise comparison of $w(T)$ and $\rho(T)$ can be made. For sample A, the magnetic field was applied parallel to the current direction, in the basal plane of the tetragonal structure: $J \| a$ and $H \perp c$; for sample B: $J \| a$ and $H \| c$.

\section{RESULTS}

Figure 2 shows $w(T)$ and $\rho(T)$ below $1 \mathrm{~K}$, for $H \perp c$ and $H \| c$, at four different values of the applied field. In all cases, both resistivities show a linear $T$ dependence, a standard signature of NFL behavior, typical of systems close to an antiferromagnetic QCP [26,27]. But in contrast to the archetypal behavior whereby the linear $T$ dependence would persist down to $T=0$ at the QCP [28], the linear $T$ dependence of $w(T)$ and $\rho(T)$ in $\mathrm{YbRh}_{2} \mathrm{Si}_{2}$ ends at a finite temperature $T^{\star}$. In Fig. 3, the downward deviation of $w(T)$ and $\rho(T)$ below $T^{\star}$ is highlighted by subtracting the linear background.

Below $T^{\star}, w(T)$ drops more rapidly than $\rho(T)$, in such a way that $w(T)$ converges towards $\rho(T)$ as $T \rightarrow 0$. This is seen most clearly in Fig. 4, where we zoom on the raw data at low temperature. A direct comparison with previously reported data, shown in Fig. 4 for $H=H_{c}$, shows that our data are in good agreement with the data of Pfau et al. [19] for $H \perp c$ 

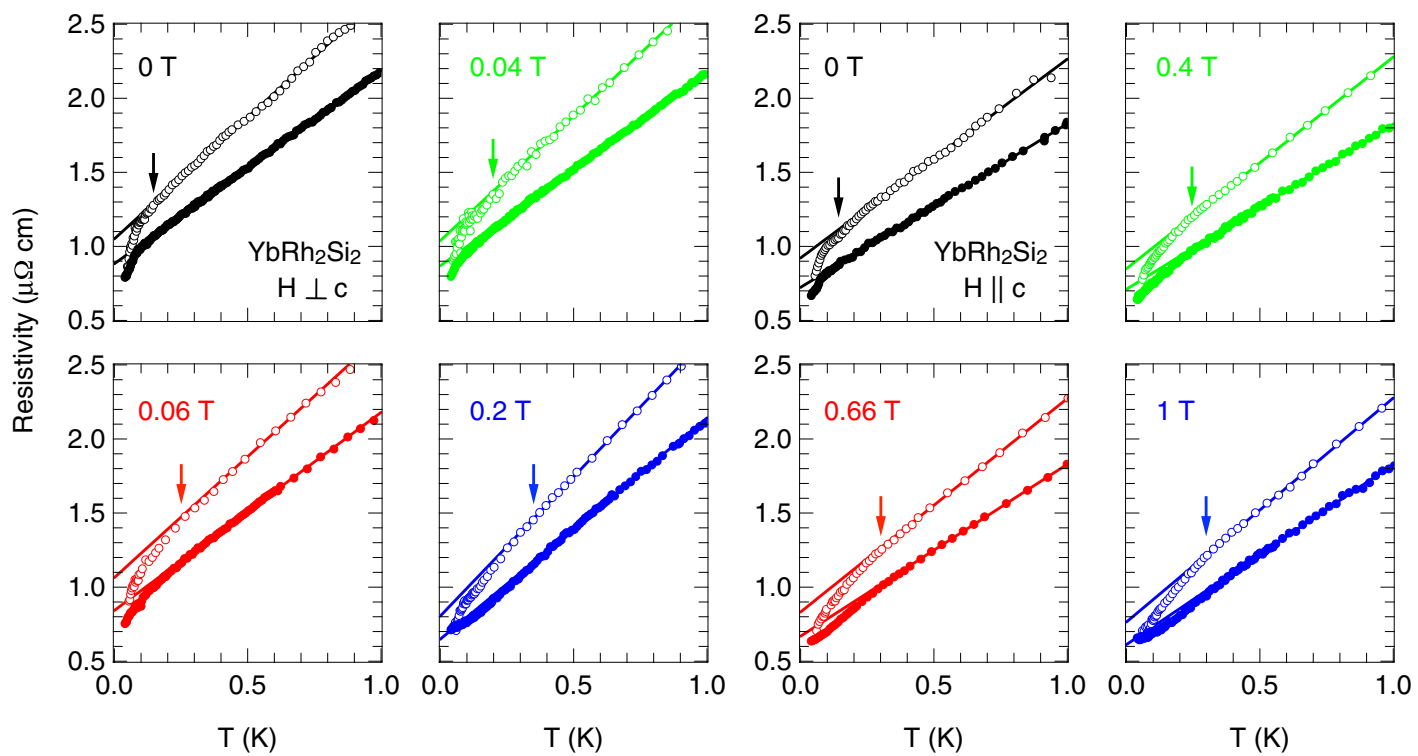

FIG. 2. (Color online) Temperature dependence of the electrical resistivity ( $\rho$; closed circles) and thermal resistivity $\left(w \equiv L_{0} T / \kappa\right.$; open circles) of $\mathrm{YbRh}_{2} \mathrm{Si}_{2}$, for currents in the basal plane $(J \| a)$. In the four panels on the left, data from sample A were obtained with a magnetic field $H \perp c$, for different field strengths as indicated. In the four panels on the right, data from sample B were obtained with $H \| c$. Data in red correspond to the field-tuned quantum critical point, at $H_{c} \simeq 0.06 \mathrm{~T}$ (left; $H \perp c$ ) and $H_{c} \simeq 0.66 \mathrm{~T}$ (right; $H \| c$ ), respectively. Above a characteristic temperature $T^{\star}$ (arrow), $w(T)$ and $\rho(T)$ are both linear in temperature; below $T^{\star}$, they both deviate downward, and converge as $T \rightarrow 0 . T^{\star}$ remains finite even at $H_{c}$.

and with the data of Machida et al. [20] for $H \| c$, modulo the downward shift of our data, due to the higher quality of our samples.

It is instructive to plot the difference between thermal resistivity and electrical resistivity, $\delta(T) \equiv w(T)-\rho(T)$, as done in Fig. 5. As discussed previously [11,12,21], $\delta(T)$ reflects the degree to which inelastic scattering is more effective in degrading a heat current than a charge current. In particular, this includes small-angle scattering processes that change the energy of the carriers without affecting their momentum direction. As seen in Fig. 5, the $\delta(T)$ curves are essentially the same for $H$ below, at, and above $H_{c}$, for both field directions: a linear $T$ dependence down to $0.2 \mathrm{~K}$, and then a rapid dive towards zero below $0.2 \mathrm{~K}$. The dive at $H=0$ is clearly caused by antiferromagnetic order below $T_{\mathrm{N}}$ (inset of Fig. 5, left panel). We propose a related mechanism for the similar dive in $\delta(T)$ at finite $H$, namely the onset of magnetic correlations.

\section{DISCUSSION}

\section{A. Temperature scale $T^{\star}$}

Our main finding is the existence of a crossover temperature $T^{\star}$ below which $\rho(T)$ and $w(T)$ deviate from their linear $T$ dependence at higher $T$. In Fig. $6, T^{\star}$ is plotted in the $H-T$ diagram of $\mathrm{YbRh}_{2} \mathrm{Si}_{2}$, for $H \| c$. It traces a line that rises smoothly from $T^{\star} \simeq T_{\mathrm{N}}$ at $H=0$ to $T^{\star} \simeq 0.35 \mathrm{~K}$ at $H=H_{c}$
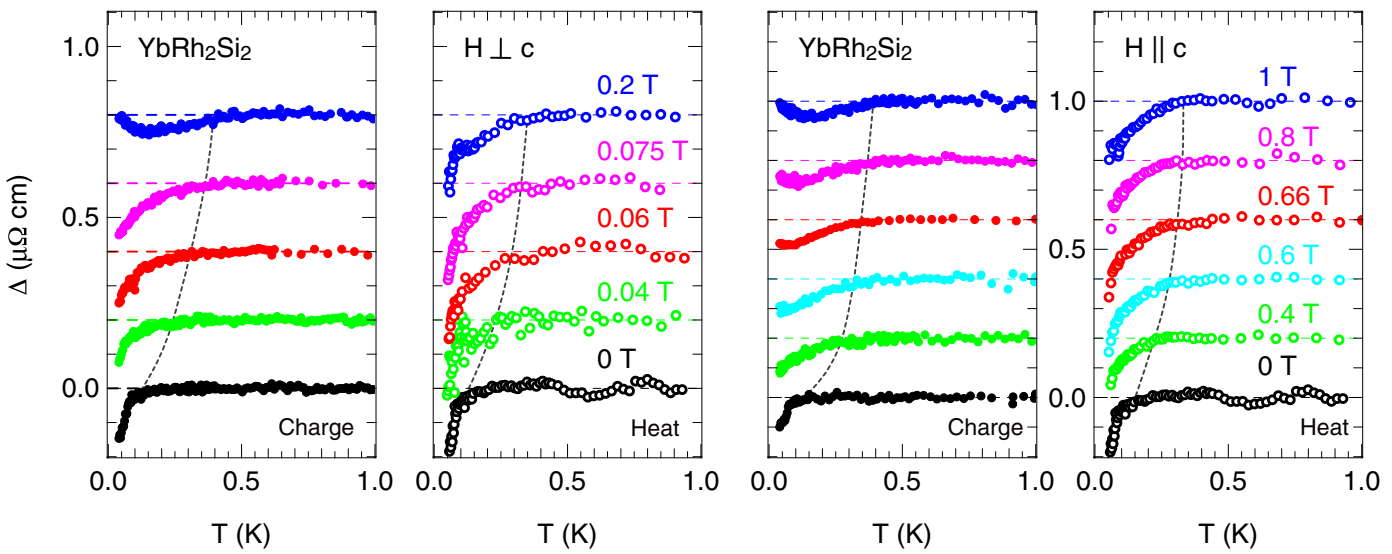

FIG. 3. (Color online) Electrical resistivity $\rho(T)$ and thermal resistivity $w(T)$ as a function of temperature, for field directions and strengths as indicated. A linear fit has been subtracted from the raw data, so that the quantities being plotted are $\Delta(T)=\rho_{\text {fit }}-\rho(T)$ (charge; closed circles) and $\Delta(T)=w_{\text {fit }}-w(T)$ (heat; open circles), where $\rho_{\text {fit }}$ and $w_{\text {fit }}$ are a linear fit to $\rho(T)$ and $w(T)$, respectively, between $T=T^{\star}$ and $T=1.0 \mathrm{~K}$. The two panels on the left show data from sample A (with $H \perp c$ ); the two panels on the right show data from sample B (with $H \| c$ ). In all curves, a downward deviation in $\rho(T)$ and $w(T)$ occurs below $T^{\star}$ (black dashed line). 

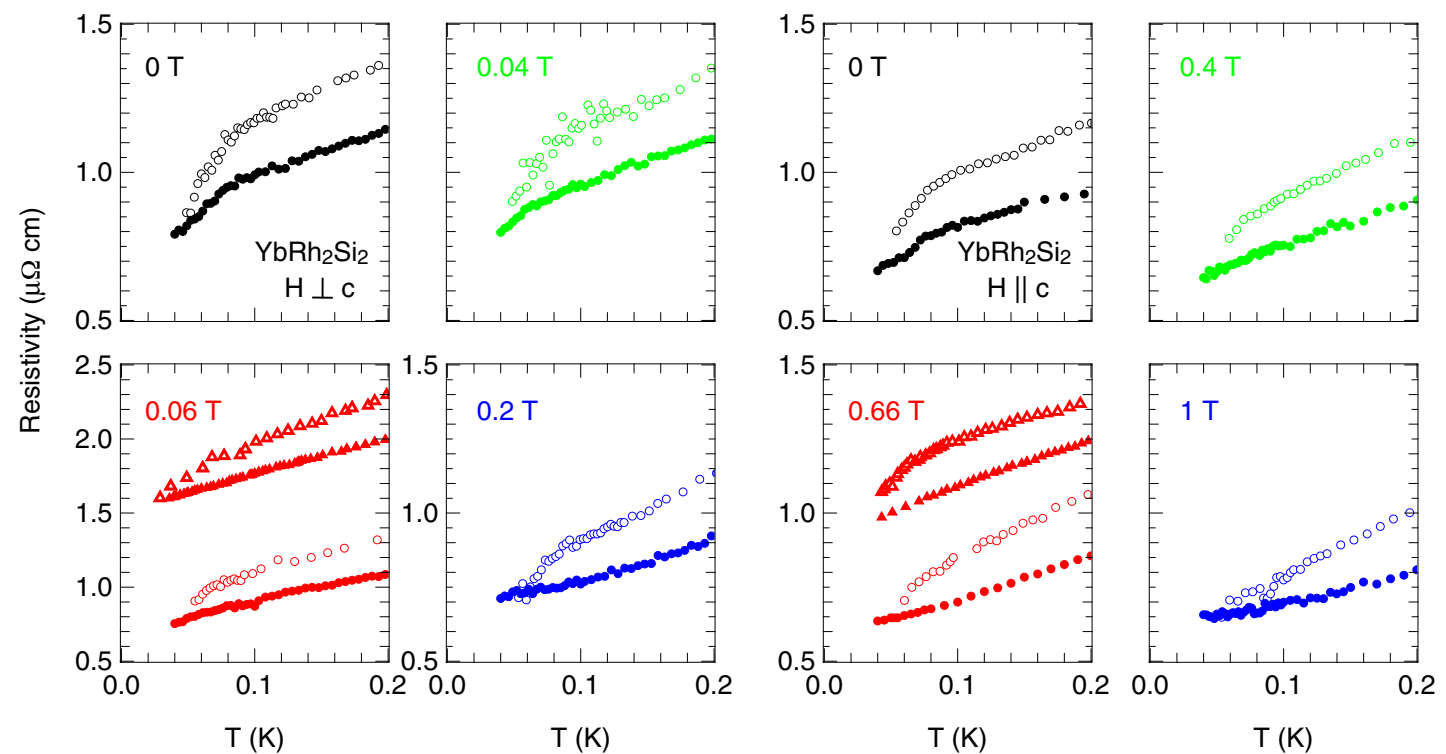

FIG. 4. (Color online) Zoom on the data of Fig. 2 below $0.2 \mathrm{~K}$. With decreasing temperature, the thermal resistivity $w(T)$ is seen to drop towards $\rho(T)$ for both field directions and all field strengths, causing the two resistivities to converge as $T \rightarrow 0$. The raw data of Pfau et al. (Ref. [19]) (red triangles; $H \perp c$ ) and Machida et al. (Ref. [20]) (red triangles; $H \| c$ ) are displayed for comparison, at $H=H_{c}$. Since $w=\rho$ as $T \rightarrow 0$ in all cases, the raw data satisfy the Wiedemann-Franz law for all values of $H$, in both field directions.

and beyond. A very similar line exists in the phase diagram for $H \perp c$. The presence of such a crossover line frames any description of the electronic behavior in $\mathrm{YbRh}_{2} \mathrm{Si}_{2}$.

Above the $T^{\star}$ line, the transport properties of $\mathrm{YbRh}_{2} \mathrm{Si}_{2}$ exhibit the linear- $T$ resistivity typical of the NFL behavior observed in the vicinity of a QCP where AF order ends [27,29]. For this regime, it is believed that the entire Fermi surface becomes "hot," in the sense that there is intense AF scattering for all wave vectors, on all parts of the Fermi surface. Indeed, a linear- $T$ electrical resistivity is observed on the border of $\mathrm{AF}$ order in the single-band quasi-1D organic superconduc- tor (TMTSF) $)_{2} \mathrm{PF}_{6}$ (Ref. [26]) and the multiband quasi-2D pnictide superconductors $\mathrm{Ba}\left(\mathrm{Fe}_{1-x} \mathrm{Co}_{x}\right)_{2} \mathrm{As}_{2}$ (Ref. [26]) and $\mathrm{BaFe}_{2}\left(\mathrm{As}_{1-x} \mathrm{P}_{x}\right)_{2}$ (Ref. [30]). It is also observed at the QCP for stripe order - a pattern of unidirectional charge and spin modulations - in cuprates $[27,28]$. In the heavy-fermion metal $\mathrm{CeCoIn}_{5}$, both $\rho$ and $w$ were shown to display linear- $T$ behavior at the field-tuned QCP [12]. This QCP is attributed to an underlying AF phase [31] hidden by the intervening superconductivity $[9,10]$.

In $\mathrm{YbRh}_{2} \mathrm{Si}_{2}$ at $H=0$, the two resistivities, and their difference, all drop abruptly below $T_{\mathrm{N}} \simeq 80 \mathrm{mK}$. A sharp drop
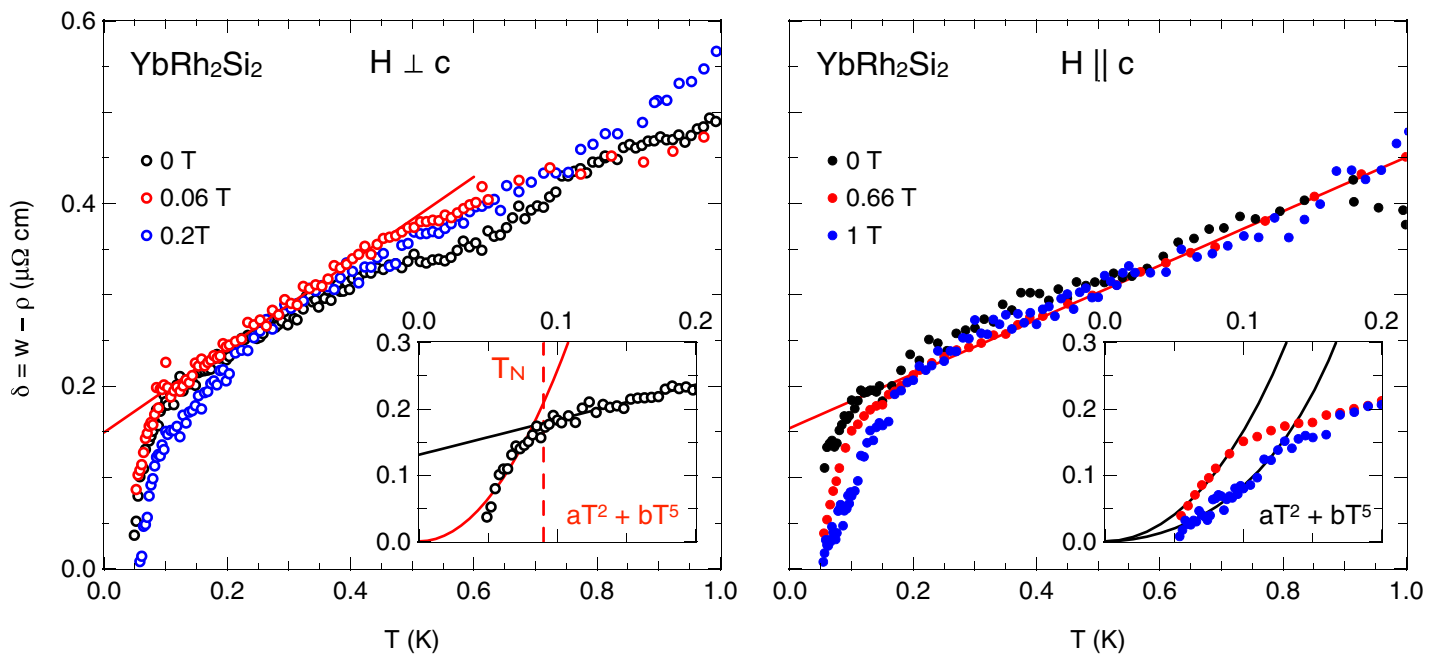

FIG. 5. (Color online) Difference between the thermal and electrical resistivities of $\mathrm{YbRh}_{2} \mathrm{Si}_{2}, \delta(T) \equiv w(T)-\rho(T)$, as a function of temperature, for $H \perp c$ (left) and $H \| c$ (right). Data are plotted for three values of $H$, as indicated. The red lines are a linear fit to the data at $H_{c}$, between 0.2 and $0.5 \mathrm{~K}$. Insets: Zoom below $0.2 \mathrm{~K}$. The vertical dotted line marks $T_{\mathrm{N}}($ at $H=0)$. For illustrative purposes, we plot the function $\delta(T)=a T^{2}+b T^{5}$ (solid curves), which provides a good description of $\delta(T)$ in the antiferromagnetic heavy-fermion metal CeRhIn ${ }_{5}$ below $T_{\mathrm{N}}$ (see Ref. [21]). This is an example of how $\delta(T)$ could rapidly converge to zero to obey the WF law. 


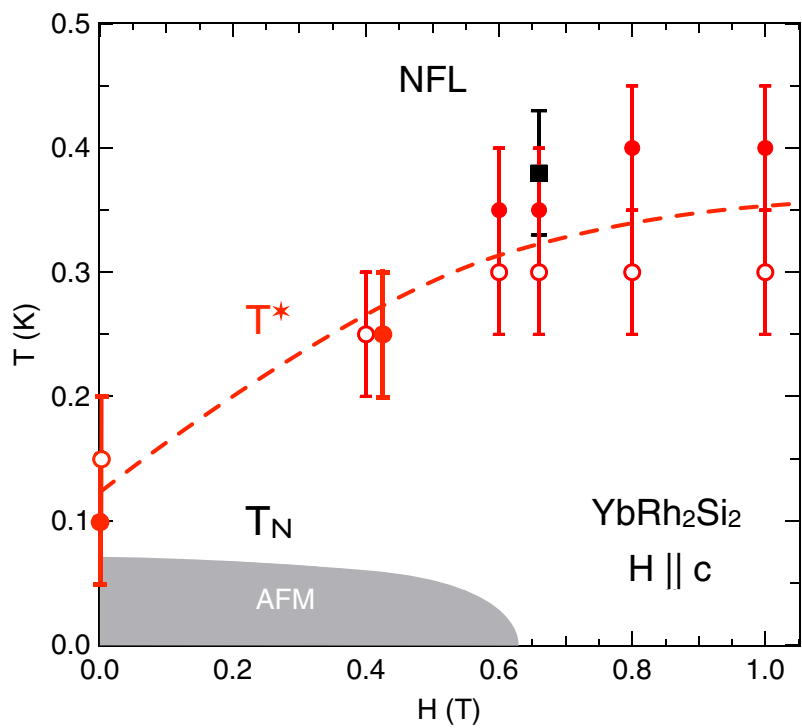

FIG. 6. (Color online) Magnetic-field-temperature phase diagram of $\mathrm{YbRh}_{2} \mathrm{Si}_{2}$, for $H \| c$. (The phase diagram for $H \perp c$ is very similar.) The region of long-range antiferromagnetic order is sketched in gray, delineated by the Néel temperature $T_{\mathrm{N}}$. The crossover temperature $T^{\star}$ below which the resistivity deviates downward from its linear $T$ dependence at high temperature is shown for heat transport (open red circles) and charge transport (closed red circles). The black square is $T^{\star}$ obtained from resistivity data in Ref. [22], for comparison. The NFL regime of linear- $T$ resistivity is confined to $T>T^{\star}$, a temperature scale that does not vanish at the quantum critical point $H_{c}=0.66 \mathrm{~T}$. We interpret $T^{\star}$ as the onset of magnetic correlations (see text).

in $\rho(T), w(T)$, and $\delta(T)$ is also observed in the heavy-fermion material CeRhIn , an antiferromagnet with $T_{\mathrm{N}}=3.8 \mathrm{~K}[21]$. Clearly, for both materials, the scattering is suppressed when the long-range magnetic order sets in, at least on some parts of the Fermi surface, which we call "cold" spots or regions.

Increasing the magnetic field applied to $\mathrm{YbRh}_{2} \mathrm{Si}_{2}$ causes the onset temperature $T_{\mathrm{N}}$ for long-range $\mathrm{AF}$ order to go to zero at $H_{c}$. However, the temperature scale $T^{\star}$ does not go to zero, but rises instead, to reach a value at $H_{c}$ which is roughly 2-3 times the zero-field value of $T_{\mathrm{N}}$ (see Fig. 6). Now the resistivity data at $H>0$ are very similar to those at $H=0$. Indeed, the in-field $\rho(T)$ and $w(T)$ drop below $T^{\star}$ in a way that is remarkably similar to the drop in the zero-field $\rho(T)$ and $w(T)$ below $T_{\mathrm{N}}$ (see Figs. 2 and 4). The difference $\delta(T)$ also behaves in a similar way at $H=0$ and $H>0$ (Fig. 5). The rapid drop in $\delta(T)$ at low temperature is roughly consistent with the drop seen in CeRhIn 5 below its $T_{\mathrm{N}}$ [21], which is well described by the function $\delta(T)=a T^{2}+b T^{5}$ (see insets of Fig. 5). Since the downward deviations in $\rho(T), w(T)$, and $\delta(T)$ for $H=0$ are due to long-range AF order, we infer that the similar but more gradual deviations seen for $H>0$ are due to short-range magnetic order.

One possibility is that $T^{\star}$ is the temperature where the thermal de Broglie wavelength, $\xi_{\text {th }} \equiv \hbar v_{\mathrm{F}} / \pi k_{\mathrm{B}} T$, becomes equal to the AF correlation length, $\xi_{\mathrm{AF}}$. In other words, when $T<T^{\star}, \xi_{\text {th }}<\xi_{\mathrm{AF}}$, so that there is AF order on the electron's length scale. Using $v_{\mathrm{F}}=2000 \mathrm{~m} / \mathrm{s}$ [32], we get $\xi_{\text {th }}=16 \mathrm{~nm}$ at $T=0.3 \mathrm{~K}$, implying that $\xi_{\mathrm{AF}} \simeq 40 a_{0}$ at $T^{\star}$, where $a_{0}$ is the in-plane lattice constant.
Two observations are consistent with short-range magnetic order developing in $\mathrm{YbRh}_{2} \mathrm{Si}_{2}$ at $H=H_{c}$ below $T^{\star} \simeq 0.35 \mathrm{~K}$. The specific heat exhibits an upward deviation from its $\log (1 / T)$ NFL dependence below $T \simeq 0.3 \mathrm{~K}[17,33]$, and the magnetic susceptibility obeys a Curie-Weiss law, with a Curie-Weiss temperature of $-0.32 \mathrm{~K}$ [16]. In other words, the presence of short-range order at $H_{c}$ prevents the NFL behavior in $\mathrm{YbRh}_{2} \mathrm{Si}_{2}$ from extending down to $T=0$ at the QCP.

The NFL behavior above some weakly field-dependent temperature scale and the recovery of "conventional" quasiparticle physics at low temperature are reproduced by a simple model of a Zeeman-driven Lifshitz transition in $\mathrm{YRh}_{2} \mathrm{Si}_{2}$ [34]. Another possible interpretation for the nonvanishing temperature scale $T^{\star} \simeq 0.3 \mathrm{~K}$ is a crossover from a regime of weakly interacting $2 \mathrm{D}$ antiferromagnetic fluctuations to a regime of strongly interacting 3D fluctuations [35].

\section{B. Wiedemann-Franz law}

In Fig. 4, we saw how $w(T)$ falls at low temperature and converges towards $\rho(T)$, for all $H$. In Fig. 5, the difference $\delta(T)$ between $w$ and $\rho$ decreases rapidly as $T \rightarrow 0$, for all $H$. In Fig. 7, we reproduce $\delta(T)$ at $H=H_{c}$ for $H \| c$. The linear $T$ dependence of the NFL regime above $T^{\star} \simeq 0.35 \mathrm{~K}$ does not persist down to $T=0: \delta(T)$ eventually deviates downwards and exhibits a rapid drop at low temperature. In Fig. 7, we

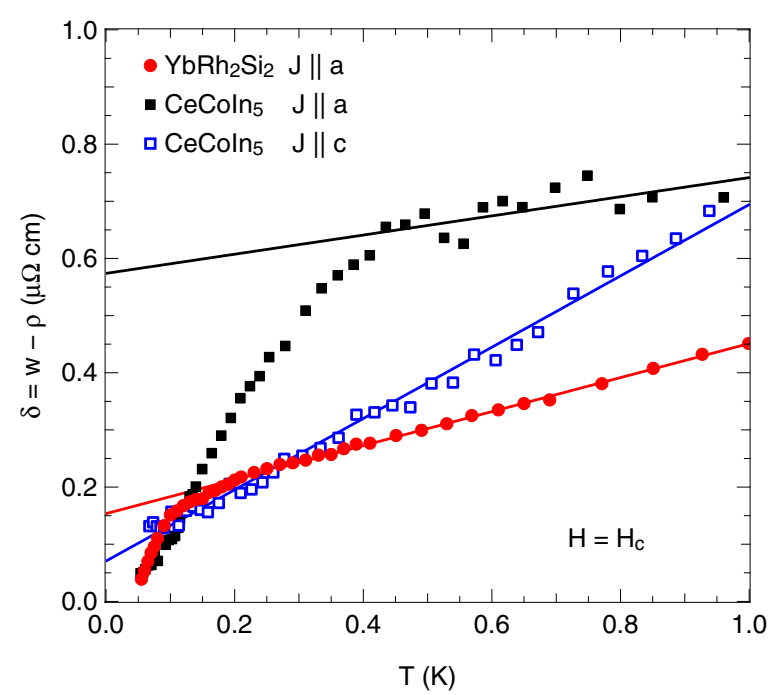

FIG. 7. (Color online) Temperature dependence of $\delta \equiv w-\rho$, the difference between thermal and electrical resistivities, for the heavy-fermion metals $\mathrm{YbRh}_{2} \mathrm{Si}_{2}$ and $\mathrm{CeCoIn}_{5}$, at the critical field $H_{c}$ of their field-tuned QCP (for $H \| c$ ), namely $0.66 \mathrm{~T}$ and $5.3 \mathrm{~T}$, respectively. The current direction is $J \| a$ for $\mathrm{YbRh}_{2} \mathrm{Si}_{2}$ (red circles), and either $J \| a$ (full black squares) or $J \| c$ (open blue squares) for $\mathrm{CeCoIn}_{5}$ (data from Ref. [12]). Solid lines are a linear fit to the high-temperature data. For $J \| a, \delta(T)$ in both materials falls at low temperature, so that the Wiedemann-Franz law is satisfied, namely $\delta(T) \rightarrow 0$ at $T \rightarrow 0$. In both cases, the fall occurs well below the temperature scale $T^{\star}$, interpreted as the onset of short-range magnetic order. By contrast, for $J \| c$ in $\mathrm{CeCoIn}_{5}$, there is no finite $T^{\star}$ and $\delta(T)$ is seen to retain its linear temperature dependence down to the lowest temperature, so that the WF law is violated in this direction (Ref. [12]). 
see how $\delta(T)$ in $\mathrm{CeCoIn}_{5}$ and $\mathrm{YbRh}_{2} \mathrm{Si}_{2}$ are very similar (for $J \| a): \delta(T)$ is linear at high temperature and it drops at low temperature, below $T \simeq 0.4 \mathrm{~K}$ in the former and below $T \simeq$ $0.1 \mathrm{~K}$ in the latter. $\mathrm{InCeCoIn}_{5}, \delta(T)$ clearly vanishes as $T \rightarrow 0$, showing that $w=\rho$ at $T=0$, satisfying the WF law. By analogy, we infer that in the limit of $T=0$ the WF law is also satisfied in $\mathrm{YbRh}_{2} \mathrm{Si}_{2}$.

In both materials (for $J \| a$ ), the linear $T$ dependence of $\rho(T)$ at $H_{c}$ is cut off at a finite $T^{\star}$, and then, at a temperature well below $T^{\star}, \delta(T)$ starts its rapid drop to zero. We associate the recovery of the WF law at $T \rightarrow 0$ with the preceding onset of short-range magnetic order at $T^{\star}$. The AF correlations would cause a $k$-dependent reduction of scattering, so that "cold" regions appear on the Fermi surface below $T^{\star}$.

By contrast, when $J \| c, \rho(T)$ in $\mathrm{CeCoIn}_{5}$ remains perfectly linear down to the lowest measured temperature $(50 \mathrm{mK})$ and $w(T)$ is also linear all the way down [12]. So unlike for $J \| a$, there is no finite temperature scale, and $T^{\star} \simeq 0$ (or at least $\left.T^{\star}<0.05 \mathrm{~K}\right)$. And for that current direction $(J \| c), \delta(T)$ retains its linear $T$ dependence all the way down. This is true not only at $H_{c}$ (see Fig. 7), but also at fields away from $H_{c}$ (see Fig. S2 in Ref. [12]). In that context, the extrapolation to $T=0$ is unambiguous. Away from the QCP, at $H=10$ $\mathrm{T} \simeq 2 H_{c}$, the extrapolation yields $\delta(0)=0$, showing the WF law to be cleanly satisfied. As $H \rightarrow H_{c}$, the entire $\delta(T)$ curves shift rigidly upwards, causing $\delta(0)$ to become nonzero, rising gradually to reach a maximum value of $\delta(0) \simeq 0.1 \mu \Omega \mathrm{cm}$ at $H_{c}=5.3 \mathrm{~T}$ [12]. Therefore, in $\mathrm{CeCoIn}_{5}$, the violation of the WF law is tuned by the field, cleanly and gradually. This is not the case in $\mathrm{YbRh}_{2} \mathrm{Si}_{2}$ : the $\delta(T)$ curves in Fig. 5 at $H<H_{c}, H=H_{c}$, and $H>H_{c}$ are not shifted relative to each other, above $T \simeq 0.2 \mathrm{~K}$, i.e., in the range from which $\delta(0)$ is extrapolated. This means that if there really is a nonzero $\delta(0)$ that violates the WF law in $\mathrm{YbRh}_{2} \mathrm{Si}_{2}$, then it is not tuned by the magnetic field (in either orientation), at least in the range up to $H \simeq(2-3) H_{c}$.

The strong anisotropy of transport in $\mathrm{CeCoIn}_{5}$ confirms that the scattering mechanism in that material—presumably AF spin fluctuations-is strongly anisotropic, or $k$ dependent. It would be interesting to see whether the same is true in $\mathrm{YbRh}_{2} \mathrm{Si}_{2}$, by performing transport measurements for $J \| c$.

We stress that our data on $\mathrm{YbRh}_{2} \mathrm{Si}_{2}$ are in excellent agreement with those of Pfau et al. [19] and Machida et al. [20] (see Fig. 4, for example). So the conclusion reached by Pfau et al. that the WFL is violated (in the sense that $L / L_{0} \rightarrow 0.9$ as $T \rightarrow 0$ at $H_{c}$, for $H \perp c$ ) is not based on a difference in the data, but rather on their assumption that there is a significant contribution from paramagnons, so that $\kappa=$ $\kappa_{\text {electron }}+\kappa_{\text {paramagnon }}$. Subtracting this putative contribution $\left(\kappa_{\text {paramagnon }}\right)$ would restore the linear $T$ dependence of $\delta(T)$ so that it would extrapolate to $\delta=0.15$ at $T \rightarrow 0$ (see Fig. 5). Like Machida et al., we disagree with this assumption. One reason is that any paramagnon contribution should presumably decrease as one moves away from the QCP. However, the drop in $\delta(T)$ does not diminish with increasing field above $H_{c}$. At $H=3 H_{c}(H \perp c)$, the drop is as pronounced as at $H_{c}$ (Fig. 5, left panel). At $H=7 H_{c}(H \| c)$, the drop in $\delta(T)$ is still very strong [20].

One way to experimentally test whether the WF violation claimed by Pfau et al. is real is to investigate how the nonzero
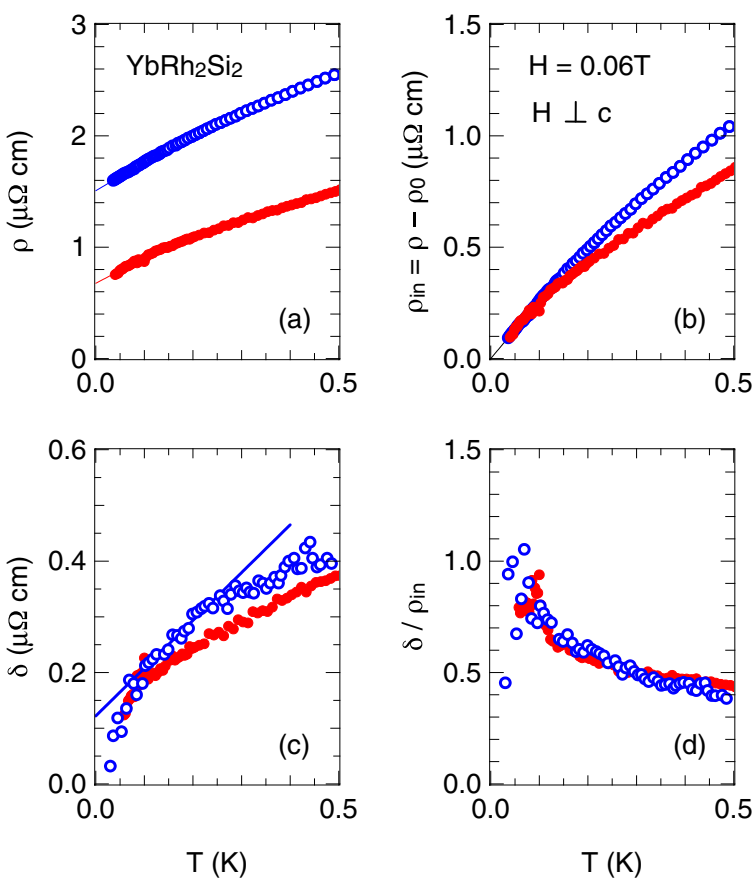

FIG. 8. (Color online) Comparison of $\rho$ and $\delta$ in two samples of $\mathrm{YbRh}_{2} \mathrm{Si}_{2}$ with different levels of disorder, at $H=H_{c}=0.06 \mathrm{~T}$ ( $H \perp c$ ). Our sample (A; full red dots) has a $\rho_{0}$ value that is roughly half that of the sample used by Pfau et al. (Ref. [19]) (No. 1; open blue circles). (a) This factor 2 difference in elastic scattering causes a large shift in $\rho(T)$. (b) By subtracting the elastic term $\rho_{0}$, we obtain the purely inelastic contribution to $\rho(T)$, namely $\rho_{\text {in }}(T) \equiv \rho(T)-\rho_{0}$. (c) The question is whether $\delta(T) \equiv \delta_{\text {in }}(T)+\delta_{0}$ is purely inelastic or not, i.e., whether $\delta_{0}=0$ or not. Pfau et al. proposed that the right way to extrapolate $\delta(T)$ is as shown by the solid line, yielding $\delta_{0} \simeq 0.13 \mu \Omega \mathrm{cm}$ (Ref. [19]). (d) Ratio of $\delta(T)$ to $\rho_{\text {in }}(T)$. The fact that this ratio is exactly the same for the two samples over the full temperature interval shows that $\delta(T)$ is entirely inelastic, just as $\rho_{\text {in }}(T)$ is; i.e., $\delta_{0}=0$.

$\delta(0)$ extrapolated from above the downturn depends on the level of elastic impurity scattering, i.e., how the apparent violation depends on $\rho_{0}$. In Fig. 8, we compare the transport properties of two samples with different levels of disorder, namely our sample A and sample No. 1 from Pfau et al., with $\rho_{0}$ values that differ by a factor 2 . Subtracting $\rho_{0}$ from $\rho(T)$ gives us the purely inelastic resistivity, $\rho_{\text {in }}(T) \equiv \rho(T)-\rho_{0}$, plotted for the two samples in Fig. 8(b). There is little ambiguity in how to extrapolate $\rho(T)$ to $T=0$, to get $\rho_{0}$, and the resulting $\rho_{\text {in }}$ does indeed satisfy the condition that $\rho_{\text {in }}(T \rightarrow 0)=0$.

The question is whether $\delta(T)$ is also purely inelastic, in which case it would satisfy the WF law, or whether it in fact contains a nonzero residual term $\delta(0)$, in which case it would violate the WF law. In other words, is $\delta_{0}=0$ in $\delta(T) \equiv \delta_{\text {in }}(T)+\delta_{0}$, or is $\delta_{0}>0$. As shown in Fig. 8(c), if one extrapolates $\delta(T)$ to $T=0$ from a linear fit above $T=0.10 \mathrm{~K}$, as illustrated for the data of Pfau et al., then one indeed gets a nonzero value, namely $\delta(T \rightarrow 0) \simeq 0.13 \mu \Omega \mathrm{cm}$ [19].

In Fig. 8(d), we directly compare the measured $\delta(T)$ with the purely inelastic part of the resistivity, $\rho_{\text {in }}(T)$, for each sample. We see that the ratio $\delta / \rho_{\text {in }}$ is identical in the two 
samples, over the entire temperature interval, despite the large difference in disorder scattering. This very strongly suggests that $\delta(T)$ is also purely inelastic. Indeed, if it were not so, i.e., if $\delta_{0}$ were not zero, then the ratio $\delta / \rho_{\text {in }}$ would diverge as $T \rightarrow 0$, and it would do so differently for the two samples (since the $\delta_{0}$ values would be different, reflecting the difference in $\rho_{0}$ ). There is no indication of any divergence or any difference. We conclude that the weight of evidence is against a violation of the WF law in $\mathrm{YbRh}_{2} \mathrm{Si}_{2}$.

It is interesting to consider the magnitude of the inelastic Lorenz ratio, defined as $L_{\text {in }} / L_{0} \equiv \rho_{\text {in }} / w_{\text {in }}$. If we assume that $\delta$ is entirely electronic, then $L_{\text {in }} / L_{0}=1 /\left(1+\delta / \rho_{\text {in }}\right)$. In $\mathrm{YbRh}_{2} \mathrm{Si}_{2}$, the data of Fig. 8(d) then yield $L_{\text {in }}(T) / L_{0} \simeq$ $0.5-0.7$, in the temperature range below $1 \mathrm{~K}$. This is very much the magnitude expected of a typical metal at low temperature, where $L_{\text {in }}(T) / L_{0} \simeq 0.4-0.6$ [11]. For example, in the heavy-fermion metals $\mathrm{CeRhIn}_{5}$ and $\mathrm{UPt}_{3}, L_{\text {in }} / L_{0}=0.4$ and 0.65 , respectively [11]. This strengthens the case for a purely inelastic $\delta$ in $\mathrm{YbRh}_{2} \mathrm{Si}_{2}$, i.e. that $\delta(0)=0$.

\section{SUMMARY}

To summarize, we have measured heat and charge transport across the magnetic-field-tuned phase diagram of $\mathrm{YbRh}_{2} \mathrm{Si}_{2}$ for fields both parallel and perpendicular to the $c$ axis. For a current in the basal plane, the thermal and electrical resistivities exhibit a linear temperature dependence, characteristic of the non-Fermi-liquid behavior found in the vicinity of an antiferromagnetic quantum critical point. However, we find that this non-Fermi-liquid behavior does not extend down to $T=0$, even at the critical field where the long-range AF order in $\mathrm{YbRh}_{2} \mathrm{Si}_{2}$ vanishes. The linear- $T$ regime ends at a characteristic temperature $T^{\star}$, below which the thermal and electrical resistivities fall and converge as $T \rightarrow 0$. Based on the similarity between transport signatures of $T_{\mathrm{N}}$ at $H=0$ and signatures of $T^{\star}$ at $H>0$ we infer that $T^{\star}$ marks the onset of short-range magnetic order. This short-range order prevents the NFL behavior from persisting down to $T=0$. The phenomenology is similar to that observed at the field-tuned quantum critical point of the heavy-fermion metal $\mathrm{CeCoIn}_{5}$.

The existence of a finite temperature scale $T^{\star}$ in $\mathrm{YbRh}_{2} \mathrm{Si}_{2}$ and $\mathrm{CeCoIn}_{5}$ raises the possibility of a precursor regime above the onset of long-range antiferromagnetic order, at $T_{\mathrm{N}}$. An interesting example of this occurs in the iron arsenide $\mathrm{BaFe}_{2} \mathrm{As}_{2}$ doped with $\mathrm{Co}$, where the resistivity deviates from its linear $T$ dependence below a temperature $T^{\star}$ that can be as high as $2 T_{\mathrm{N}}$ [36].

Finally, by comparing samples of different residual resistivity, we argued that the Wiedemann-Franz law is satisfied in $\mathrm{YbRh}_{2} \mathrm{Si}_{2}$ at the quantum critical point for $H \perp c$, and we infer that it is most likely satisfied at all fields and for both directions.

\section{ACKNOWLEDGMENTS}

We thank K. Behnia, J.-P. Brison, P. Coleman, J. Custers, J. Flouquet, P. Gegenwart, S. Hartnoll, R. W. Hill, S. R. Julian, J. Paglione, F. Ronning, T. Senthil, and F. Steglich for insightful discussions, and J. Corbin for his assistance with the experiments. The work at Sherbrooke was supported by the Canadian Institute for Advanced Research and a Canada Research Chair and it was funded by NSERC, FQRNT, and CFI. Part of the work was carried out at the Brookhaven National Laboratory, which is operated for the US Department of Energy by Brookhaven Science Associates (DE-Ac02-98CH10886) and in the Ames Laboratory, supported by the US Department of Energy, Office of Basic Energy Sciences, Division of Materials Sciences and Engineering, under Contract No. DE-AC02$07 \mathrm{CH} 11358$.
[1] N. D. Mathur, F. M. Grosche, S. R. Julian, I. R. Walker, D. M. Freye, R. K. W. Haselwimmer, and G. G. Lonzarich, Nature (London) 394, 39 (1998).

[2] P. Coleman and A. J. Schofield, Nature (London) 433, 226 (2005).

[3] T. Senthil, M. Vojta, and S. Sachdev, Phys. Rev. B 69, 035111 (2004).

[4] P. Coleman, J. B. Marston, and A. J. Schofield, Phys. Rev. B 72, 245111 (2005).

[5] D. Podolsky, A. Vishwanath, J. Moore, and S. Sachdev, Phys. Rev. B 75, 014520 (2007).

[6] K.-S. Kim and C. Pépin, Phys. Rev. Lett. 102, 156404 (2009).

[7] W. Witczak-Krempa, P. Ghaemi, T. Senthil, and Y. B. Kim, Phys. Rev. B 86, 245102 (2012).

[8] R. Mahajan, M. Barkeshli, and S. A. Hartnoll, Phys. Rev. B 88, 125107 (2013).

[9] J. Paglione, M. A. Tanatar, D. G. Hawthorn, E. Boaknin, R. W. Hill, F. Ronning, M. Sutherland, L. Taillefer, C. Petrovic, and P. C. Canfield, Phys. Rev. Lett. 91, 246405 (2003).

[10] A. Bianchi, R. Movshovich, I. Vekhter, P. G. Pagliuso, and J. L. Sarrao, Phys. Rev. Lett. 91, 257001 (2003).
[11] J. Paglione, M. A. Tanatar, D. G. Hawthorn, F. Ronning, R. W. Hill, M. Sutherland, L. Taillefer, and C. Petrovic, Phys. Rev. Lett. 97, 106606 (2006).

[12] M. A. Tanatar, J. Paglione, C. Petrovic, and L. Taillefer, Science 316, 1320 (2007).

[13] S. Kambe, H. Suderow, T. Fukuhara, J. Flouquet, and T. Takimoto, J. Low Temp. Phys. 117, 101 (1999).

[14] F. Ronning, R. W. Hill, M. Sutherland, D. G. Hawthorn, M. A. Tanatar, J. Paglione, L. Taillefer, M. J. Graf, R. S. Perry, Y. Maeno, and A. P. Mackenzie, Phys. Rev. Lett. 97, 067005 (2006).

[15] J. K. Dong, Y. Tokiwa, S. L. Bud'ko, P. C. Canfield, and P. Gegenwart, Phys. Rev. Lett. 110, 176402 (2013).

[16] P. Gegenwart, J. Custers, C. Geibel, K. Neumaier, T. Tayama, K. Tenya, O. Trovarelli, and F. Steglich, Phys. Rev. Lett. 89, 056402 (2002).

[17] J. Custers, P. Gegenwart, H. Wilhelm, K. Neumaier, Y. Tokiwa, O. Trovarelli, C. Geibel, F. Steglich, C. Pépin, and P. Coleman, Nature (London) 424, 524 (2003).

[18] Q. Si, S. Rabello, K. Ingersent, and J. L. Smith, Nature (London) 413, 804 (2001).

[19] H. Pfau, S. Hartmann, U. Stockert, P. Sun, S. Lausberg, M. Brando, S. Friedemann, C. Krellner, C. Geibel, S. Wirth, 
S. Kirchner, E. Abrahams, Q. Si, and F. Steglich, Nature (London) 484, 493 (2012).

[20] Y. Machida, K. Tomokuni, K. Izawa, G. Lapertot, G. Knebel, J.-P. Brison, and J. Flouquet, Phys. Rev. Lett. 110, 236402 (2013).

[21] J. Paglione, M. A. Tanatar, D. G. Hawthorn, R. W. Hill, F. Ronning, M. Sutherland, L. Taillefer, C. Petrovic, and P. C. Canfield, Phys. Rev. Lett. 94, 216602 (2005).

[22] O. Trovarelli, C. Geibel, S. Mederle, C. Langhammer, F. M. Grosche, P. Gegenwart, M. Lang, G. Sparn, and F. Steglich, Phys. Rev. Lett. 85, 626 (2000).

[23] P. Gegenwart, Y. Tokiwa, T. Westerkamp, F. Weickert, J. Custers, J. Ferstl, C. Krellner, C. Geibel, P. Kerschl, K-H. Mueller, and F. Steglich, New J. Phys. 8, 171 (2006).

[24] G. Knebel, R. Boursier, E. Hassinger, G. Lapertot, P. G. Niklowitz, A. Pourret, B. Salce, J. P. Sanchez, I. Sheikin, P. Bonville, H. Harima, and J. Flouquet, J. Phys. Soc. Jpn. 75, 114709 (2006).

[25] E. Boaknin, R. W. Hill, C. Proust, C. Lupien, L. Taillefer, and P. C. Canfield, Phys. Rev. Lett. 87, 237001 (2001).

[26] N. Doiron-Leyraud, P. Auban-Senzier, S. René de Cotret, C. Bourbonnais, D. Jérome, K. Bechgaard, and L. Taillefer, Phys. Rev. B 80, 214531 (2009).
[27] L. Taillefer, Annu. Rev. Condens. Matter 1, 51 (2010).

[28] R. Daou, N. Doiron-Leyraud, D. LeBoeuf, S. Y. Li, F. Laliberté, O. Cyr-Choinière, Y. J. Jo, L. Balicas, J.-Q. Yan, J.-S. Zhou, J. B. Goodenough, and L. Taillefer, Nat. Phys. 5, 31 (2009).

[29] P. Monthoux, G. G. Lonzarich, and D. Pines, Nature (London) 450, 1177 (2007).

[30] S. Kasahara, T. Shibauchi, K. Hashimoto, K. Ikada, S. Tonegawa, R. Okazaki, H. Shishido, H. Ikeda, H. Takeya, K. Hirata, T. Terashima, and Y. Matsuda, Phys. Rev. B 81, 184519 (2010).

[31] L. D. Pham, Tuson Park, S. Maquilon, J. D. Thompson, and Z. Fisk, Phys. Rev. Lett. 97, 056404 (2006).

[32] R. Joynt and L. Taillefer, Rev. Mod. Phys. 74, 235 (2002).

[33] N. Oeschler, S. Hartmann, A. P. Pikul, C. Krellner, C. Geibel, and F. Steglich, Phys. B: Condens. Matter 403, 1254 (2008).

[34] A. Hackl and M. Vojta, Phys. Rev. Lett. 106, 137002 (2011).

[35] E. Abrahams and P. Wölfle, Proc. Natl. Acad. Sci. USA 109, 3238 (2012).

[36] J.-H. Chu, J. G. Analytis, K. De Greve, P. L. McMahon, Z. Islam, Y. Yamamoto, and I. R. Fisher, Science 329, 824 (2011). 